\title{
Therapeutic genome engineering: Implications for South Africa
}

\author{
J Naidoo, ${ }^{1} \mathrm{PhD} ;$ E Fok, ${ }^{1,2} \mathrm{MSc} ;$ J Scholefield, $\mathrm{PhD}^{1,3}$ \\ ${ }^{1}$ Bioengineering and Integrative Genomics Group, Next Generation Health Cluster, Council for Scientific and Industrial Research, Pretoria, South Africa \\ ${ }^{2}$ Institute for Infectious Disease and Molecular Medicine, Faculty of Health Sciences, Department of Integrative Biomedical Sciences, University of \\ Cape Town, South Africa \\ ${ }^{3}$ Department of Human Biology, Faculty of Health Sciences, University of Cape Town, South Africa
}

Corresponding author: J Scholefield (jscholefield@csir.co.za)

South Africa encompasses extraordinary genetic diversity, frequently revealing unique mutations and variations associated with disease. Despite the advances of traditional gene therapy, our understanding of causative mutations in the South African population has, for the most part, contributed to diagnostic rather than therapeutic interventions. Recent developments in genome engineering and its ease of use have released a powerful tool with which to intervene in otherwise untreatable disease. In addition, harnessing this tool for discrete genetic edits provides a mechanism by which screening of new drugs specific to our population's diversity can be accomplished. Here, we use examples of some of the most advanced genome engineering approaches to develop therapeutic strategies that would specifically affect South African individuals.

S Afr Med J 2019;109(8 Suppl 1):S54-S58. https://10.7196/SAMJ.2019.v109i8b.13850

Over the last 5 years, genome engineering has emerged as one of the most powerful molecular tools with potential therapeutic applications. Though traditional gene therapy has come a long way, as evidenced by the recent licensing of Strimvelis as a gene therapy for severe combined immunodeficiency (SCID), nonspecific insertional oncogenesis remains a concern. ${ }^{[1]}$ Genome engineering technology bypasses this issue through targeted disruption of the genome acting as GPS coordinates for the intended genetic modification at a specifically designated site. With the recent advance and ease of use of the clustered regularly interspaced short palindromic repeats (CRISPR) technology, gene editing has been catapulted to the forefront of molecular and cellular biology, and the public eye. ${ }^{[2]}$ In this review, we will highlight the general approaches to targeting disease by expanding on a few of the most successful studies to date, though this is in no way an exhaustive review of all diseases amenable to genome engineering therapies. Using these established methods, we then discuss potential approaches that could have an impact on South African health burdens, taking into account the unique genetic diversity of the diaspora.

\section{Principles of genome engineering}

The basic approach for genome engineering involves the use of specialised 'molecular scissors' called endonucleases which can be designed to target a specific sequence of genomic DNA (gDNA) and induce a double-strand break (DSB). The most frequently exploited of these are zinc finger nucleases (ZFNs), transcription activator-like effector nucleases (TALENs) and CRISPR. ${ }^{[3]}$ DSBs stimulate the cell's repair machinery, either using the non-homologous end joining (NHEJ) or homology-directed repair (HDR) processes (Fig. 1). NHEJ often leads to incorrect insertions or deletions (indels) in the DNA. In a coding region of the genome this can be exploited to permanently knock out a gene as a result of a shift in the reading frame, creating a non-functional protein, and thus, gene ablation. This offers an opportunity to deactivate the deleterious gene in disorders with gain-of-function mutations. Though relatively efficient, this does not allow for accurate repair of loss-of-function mutations. Fortunately, exploitation of the HDR pathway during cell division with the addition of a synthetic DNA template can lead to gene correction. The efficiency of this process is significantly lower than NHEJ, and it is therefore critical that design strategies take this into consideration. The development of an endonuclease-deficient variant of CRISPRassociated protein 9 (Cas9), dead Cas9 (dCas9), has allowed us to go beyond these classic genome engineering strategies which rely on the introduction of a DSB. By fusing dCas9 to transcriptional repressors (CRISRPi) ${ }^{[4]}$ or activators (CRISPRa), ${ }^{[5]}$ loss- and gain-of-function studies can be carried out independently of a DSB.

Despite the specificity of targeting, some off-target DSBs can occur, especially when using CRISPR. ZFNs and TALENs are considered to lead to fewer off-target effects across the genome. In addition, TALENs have unlimited targeting sequence ability, while CRISPR requires a recognition sequence of 'NGG' to exert its effect ${ }^{[6]}$ However the design of these endonucleases is substantially more technically challenging, and therefore they are adopted less frequently than CRISPR.

\section{Therapeutic approaches}

There exists a myriad of ways in which genome engineering can be exploited for therapeutic uses (Fig. 2). Importantly, these reflect similar strategies used in traditional gene therapy research, but will be expanded upon here by showcasing some of the most recent and impactful research.

In vivo therapeutic engineering makes use of well-established viral vector delivery, in which the endonuclease and, if necessary, the repair construct, are integrated into recombinant viruses such as adeno-associated viruses (AAVs) and lentiviral vectors. ${ }^{[7]}$ These vectors can be manipulated to restrict tropism to a specific cell type, to enhance the specificity of the endonuclease to disease-specific cellular damage. Delivery can be systemic or localised to the area where it is most required, e.g. intrastriatal or intramuscular to increase the proportion of genetically corrected cells necessary for measurable clinical improvement (Fig. 2A).

A recent study by Amoasii et al. ${ }^{[8]}$ used such a strategy to repair a mutation in the dystrophin gene in a canine model of 


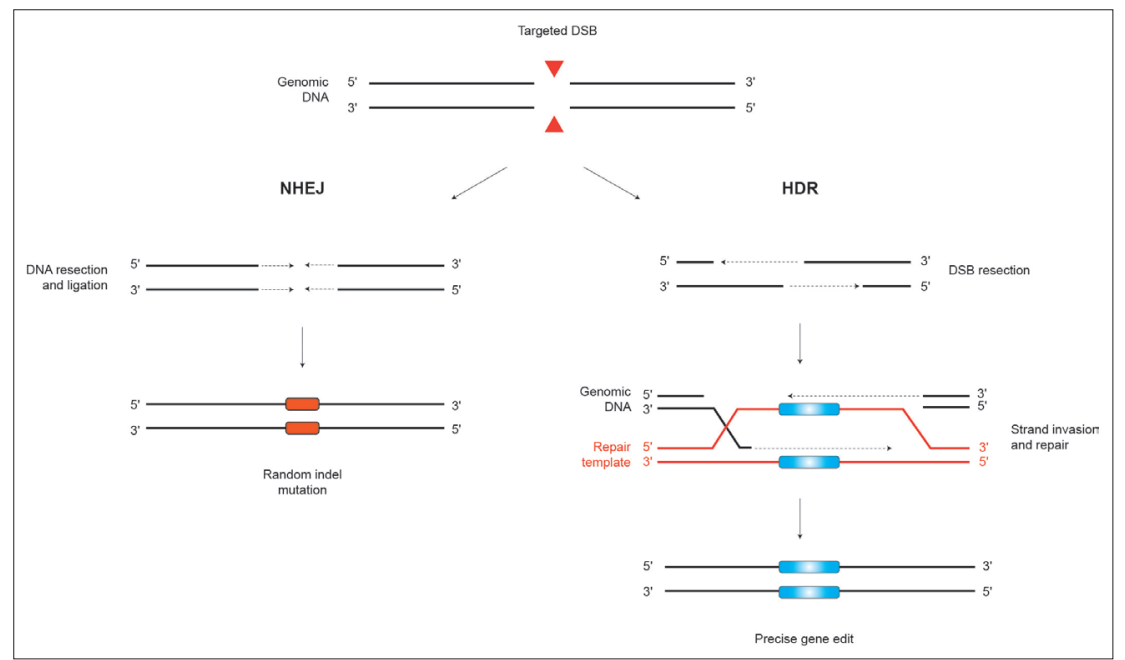

Fig. 1. Genome-editing repair mechanisms. Targeted double-strand break (DSB) results in the cellular machinery rapidly repairing the break via the error-prone non-homologous end-joining (NHEJ) errorprone pathway, which often results in insertions and deletions (indel). During mitosis, an alternative repair pathway can be employed, in the presence of a repair template (homology-directed repair (HDR)) to create a precise gene edit at the site of the DSB.

Duchenne muscular dystrophy (DMD). Many dystrophin mutations cause a frameshift of the coding region, and thus a truncated, non-functional protein leading to cardiomyopathy-induced lethality. Directly skipping exon 51 by targeting a splice-acceptor site would repair the reading frame, thereby partially restoring dystrophin function in $13 \%$ of DMD patients. This offers an 'off-the-shelf' treatment for a significant proportion of individuals. ${ }^{[9]}$ Post-mitotic cells, such as skeletal and cardiac muscle, undergo very low levels of HDR. The exploitation of NHEJ repair in terminally differentiated cells can, however, allow for skipping of a deleterious exon following DNA break induction. Despite the preliminary nature of the study, this is one of the most promising in a large animal model, demonstrating restoration of dystrophin production in all muscles after systemic injection of $1 \times 10^{14}$ AAVs9 viral particles containing CRISPRCas9 components. Importantly, the authors showed a lack of immune reaction through undetectable CD4 infiltration. Critically, both the diaphragm and cardiac muscles showed over 50\% improvement in protein levels. This successful example of in vivo genome engineering brings to the fore another crucial aspect in that the proportion of repaired cells needed to elicit meaningful phenotypic benefits is disease-specific. In DMD, it has been shown that only 15\% homogenous expression may be sufficient to slow down disease significantly in a mouse model. ${ }^{[10]}$

Another strategy of therapeutic genome engineering includes an ex vivo approach, whereby cells from an individual can be extracted, corrected in a dish, and then replaced (Fig. 2B). This offers advantages over in vivo treatments, as only corrected cells could be selected, enhancing the impact of mutation correction. This is best exemplified by FDA-approved immunotherapy treatments targeting CD19 antigens on cancer cells engineering chimeric antigen receptors on $\mathrm{T}$ cells (CART). In 2015, a 6-month-old patient was diagnosed with acute lymphoid leukaemia (ALL) with conventional treatments having failed. Using previously established CART cells, the team used TALENs to disable the $\mathrm{T}$ cell receptor alpha (TCRa) gene to prevent these cells from causing graft-versus-host disease upon infusion. ${ }^{[11]}$ Three years later, the patient has been reported to be in remission. Subsequently, CART therapy has rapidly evolved to include other blood cancers, including Kymriah (Novartis, Switzerland) for all and non-Hodgkin lymphoma, and Yescarta (Kite Pharma, USA) for diffuse large B cell lymphoma. ${ }^{[12]}$ In addition, research is now expanding these successes towards targeting solid-state tumours. ${ }^{[13]}$ However, ex vivo treatment incurs additional issues in that, while it is desirable for infused cells to proliferate and mount a strong attack on cancer cells, they can create cytokine release syndrome, which is essentially a cytokine storm leading to life-threatening side-effects. ${ }^{[14]}$ As a result, Cellectis has genetically engineered a safety switch in CART cells, yielding the foreign cells susceptible to rituximab, the FDA-approved antibody enabling control of infused cells should life-threatening side-effects emerge. ${ }^{[15]}$ These and other improvements could propel CART therapy into mainstream treatment options; however, the cost (approaching USD500 000) per treatment remains a significant challenge. Off-theshelf allogeneic treatments through the promotion of public cell banks, with standardised manufacturing pipelines, could go a long way towards a more costeffective approach. ${ }^{[16]}$

However, adult stem cells do not provide the means to make all cell types. For many neurodegenerative disorders, for example, mature neurons are needed to replace damaged cells. Pluripotent stem cells (PSCs), both embryonic stem cells (ESCs) ${ }^{[17]}$ and induced pluripotent stem cells (iPSCs), ${ }^{[18]}$ offer a unique opportunity to address this challenge. On the escarpment of Waddington's landscape, they provide a source of cellular material able to generate any cell type in the body, including a fimultitude of neuronal cell types. ${ }^{[19]}$ Importantly, iPSCs can be generated in a patient-specific manner (Fig. 2B), which negates the need for harmful immune suppressors, while incorporating gene corrections. Several studies led by the Gforce-PD consortium have made advances in cell-replacement therapies. ${ }^{[20]}$ In October 2018, Jun Takahashi and his team at Kyoto University coaxed healthy, allogeneic iPSCs into neuronal cells (precursors to dopaminergic neurons) in vitro, which were subsequently implanted into the brain of a 50-year-old Parkinson's patient. ${ }^{[21]}$ It is important to note that allogeneic matching of healthy iPSCs may bypass the need for gene correction entirely.

Isogenic PSCs further provide a method for indirect therapeutic applications. Here, genome engineering can be used to include specific mutations in separate cell lines, providing a phenomenally accurate screening tool (Fig. 2C). Researchers can use these disease-in-a-dish models to investigate the contributions of specific mutations to the disease without the significant background genetic variation, which can confound in vitro phenotypes. ${ }^{[22]}$ Similar genome engineering screening models can reveal novel targets in $\mathrm{T}$ cell immunotherapy. Patel et al. ${ }^{[23]}$ performed a CRISPR screen in $\mathrm{T}$ cells, and observed the effectiveness of individually mutated $\mathrm{T}$ cell clones in targeting melanoma cells in vitro. While these may not fall under the umbrella of cellular replacement therapy, as a tool, they provide an indirect therapeutic strategy by identifying novel pathways that 


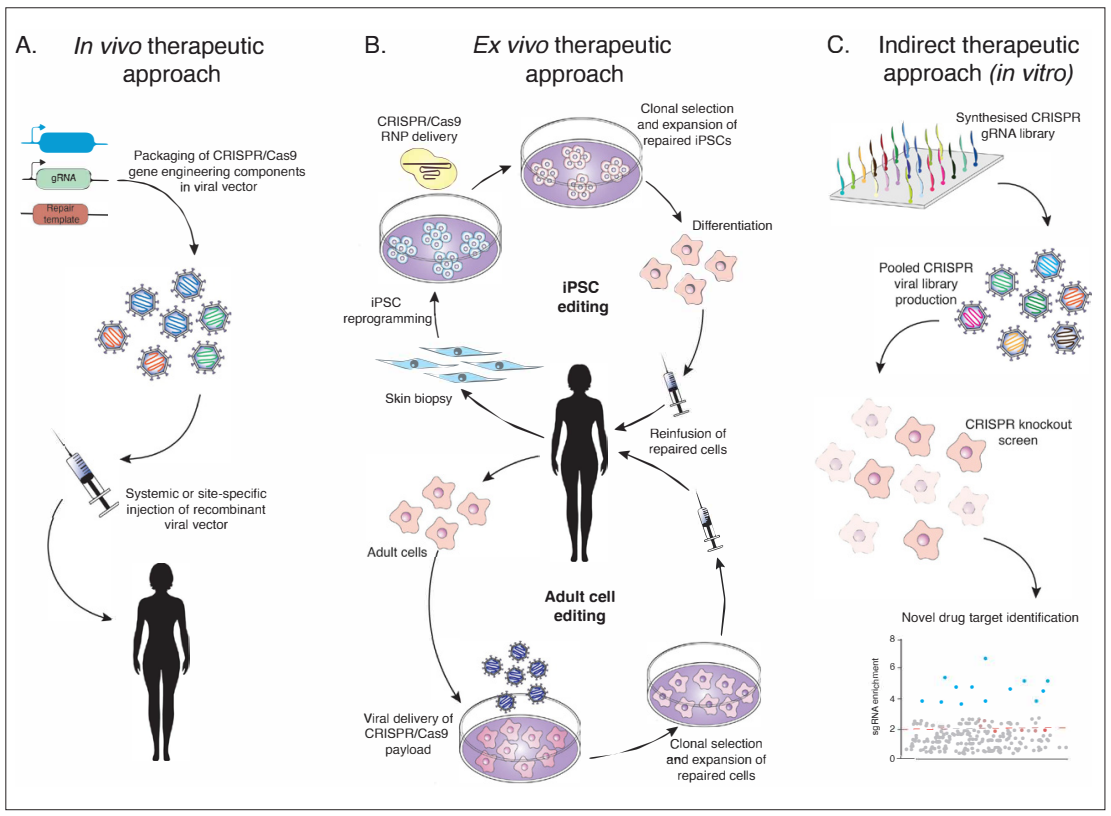

Fig. 2. An overview of the CRISPR/Cas9 therapeutic approaches. (A) In vivo therapies package the CRISPR/Cas 9 genome engineering components into viral vectors which are injected systemically or sitespecifically into a patient. The viral vectors are tuned to infect specific cell types and deliver the CRISPR/ Cas 9 payload for corrective genome engineering. (B) Ex vivo approaches involve removing the patient cells and genetically manipulating them in a tissue culture dish. This can be done directly in adult cells, or in reprogrammed iPSCs, which subsequently have to be differentiated into the cell type of interest. The corrected cells are selected for, expanded and then reinfused into the patient as an autologous transplantation. (C) Genome-wide CRISPR/Cas9 knockout screens can be used to elucidate novel drug targets by assessing drug efficacy in the context of de novo CRISPR/Cas9-induced gene mutations. $(C R I S P R=$ clustered regularly interspaced short palindromic repeats; $i P S C s=$ induced pluripotent stem cells; Cas $9=$ CRISPR-associated protein 9.)

can be targeted to improve immunotherapy outcomes.

\section{Genome engineering for South Africa}

South Africa (SA) is crippled with a high burden of infectious disease, and increasing incidences of non-communicable diseases. One may argue that we should channel our limited resources into tackling these health issues first, and allow the West to research advanced genome engineering technologies that could apply to our patients once developed. However, a 'onesize-fits-all' therapeutic approach is often not feasible because of the unique genetics and admixture of our population groups. Here, we evaluate the potential for genome engineering strategies to tackle diseases that would impact on social, genetic and economic issues pertinent or unique to the country.

\section{Infectious disease}

In 2017, SA's HIV expenditure was over USD2 billion. ${ }^{[24]}$ Notably, these life-saving treatments have also been associated with a number of adverse side-effects that are significantly more prevalent in individuals of African descent. ${ }^{[25]}$ Thus, while current ART approaches continue to play an essential role in curbing AIDS-related mortalities there is a clear need for therapeutic cures.

Gene therapy-based approaches have long represented promising avenues of research given the characterisation of rare human genetic variants that confer natural resistance against HIV. ${ }^{[26]}$ The most well characterised of these is a 32 base pair deletion in the gene encoding the chemokine receptor, C-C chemokine receptor type 5 (CCR5). This mutation, referred to as CCR5- $\Delta 32$, is most common in individuals of Northern and Eastern European descent $(\sim 10 \%)$, where a homozygous deletion of CCR5 results in complete viraemic control and heterozygosity results in delayed disease progression to AIDS. The landmark Berlin patient publication of 2009 demonstrated how an HIV-positive patient with leukaemia was cured of HIV through a haematopoietic stem-cell (HSC) transplant from an HLAmatched, CCR5- $\Delta 32$ homozygous donor. ${ }^{[27]}$

Allogeneic matching of donors may not be feasible in the highly diverse genetic populations in SA. The use of genome- editing strategies to first engineer and later reintroduce autologous cell populations may, however, overcome many of the challenges associated with donor-transplant approaches.

Numerous studies have demonstrated promising results for genome-engineering strategies in the fight against HIV/AIDS through the ablation of HIV-associated co-receptors (CCR5 and C-X-C chemokine receptor type 4 (CXCR4) ${ }^{[28]}$ and the viral regulatory proteins from infected $\mathrm{T}$ cell populations. ${ }^{[29]}$ Other studies have created second generation CART therapy to develop HIV-resistant $\mathrm{T}$ cell population $\mathrm{s}^{[30]}$ or deleted HIV promoter elements from circulating cells in patient-engrafted mice. ${ }^{[31]}$ Finally, using derivations of the CRISPR system, eradication of latent viral reservoirs through CRISPR-based activator systems has been achieved using in vitro $\mathrm{T}$ cell models of latency where a $>85 \%$ activation rate was observed. ${ }^{[32]}$ HIV further lends itself to genome engineering knockout screens and the subsequent identification of novel drug targets as suggested in Fig. 1C. ${ }^{[33]}$ At the CSIR, we have utilised CRISPRbased technology to produce iPSC-derived macrophages, incorporating biallelic CCR5 gene ablation as well as African relevant 3D hepatic models, for the evaluation of ART-associated adverse drug reactions (unpublished data). These examples of in vivo therapeutic genome engineering and indirect in vitro studies may significantly impact the crippling cost of life-long antiretroviral therapy.

Hepatitis B virus (HBV) has reliable prophylactic vaccines but no permanent cure. With inadequate vaccine deployment, chronic HBV infection leads to cirrhosis and hepatocarcinoma. HBV undergoes several stages of its life cycle but is susceptible at one particular point. Targeting of the stable episomal covalently closed circular DNA (cccDNA) of the virus provides an opportunity to permanently disable the virus, since it encodes a number of essential regions, rendering it susceptible to genetically engineered disruptions. ${ }^{[34]}$ Research into targeting HBV is being led by Arbuthnot et $a l .{ }^{[35]}$ at the University of the Witwatersrand, demonstrating one of the first uses of targeting the virus directly using TALENS. In essence, this would provide a mechanism for inhibiting the re-emergence of latent viruses. Effective approaches targeting the cccDNA of $\mathrm{HBV}$ are still reliant on efficient delivery of genome-engineering tools to hepatocytes. ${ }^{[36]}$

Many viral vectors, like AAVs, can only deliver small genomic cargo. Advances made in derivations of CRISPR-Cas9, for example 
using the smaller saCas9, will thus significantly aid curative treatments for $\mathrm{HBV}^{[37]}$

\begin{abstract}
Albinism
Albinism has a special significance in SA due in part to the climate, social discrimination and abuse experienced by patients, ${ }^{[38]}$ notwithstanding the clinical phenotype, which includes increased susceptibility to cancer and poor eyesight. ${ }^{[39]}$ In one form, as a result of a founder mutation in Africans, a mutation in the oculocutaneous albinism 2 (OCA2) gene leads to an incidence of $1 / 3900 \mathrm{SA}$ individuals. It is therefore the most common recessive disorder in SA, with $80 \%$ of patients carrying a $2.7 \mathrm{~kb}$ deletion in exon $7 .{ }^{[40]}$ The deletion leads to reduced production of a protein essential to melanin production. Given the complexity of skin structure, an ex vivo therapeutic approach involving gene correction, and subsequent transplantation of healthy melanocytes, is not currently feasible. However, genome engineering of isogenic iPSCs, could remove compounding genetic variation between individuals to exclusively compare cells with and without the mutation. Subsequent differentiation into melanocytes and retinal pigment epithelium (RPE) would then provide a screening tool with which to identify drugs that may alleviate melanin production, as is being researched for $O C A 1{ }^{[41]}$ Thus, an indirect therapeutic approach specifically for OCA2 SA patients would significantly impact the lives of these patients.
\end{abstract}

\section{Neurodegenerative disorders}

Disorders that lead to degeneration of neurons fall short of treatment options globally. While genetic research has led to discoveries of how mutations cause disease, diagnosis has largely been the only contribution towards managing symptoms without significant treatment options. Spinal muscular atrophy (SMA) is an autosomal recessive disease frequently caused by a homozygous deletion of the SMN1 gene. However, an evolutionary backup in the form of a duplication, results in the presence of the SMN2 gene that can, with increased expression, rescue the degeneration of motor neurons. ${ }^{[42]}$

A single nucleotide mutation in the SMN2 gene prevents correct splicing and thus expression of SMN protein from this backup gene. Targeting this small genetic change is a recently approved FDA and EMA treatment, which uses intrathecal delivery of an antisense oligonucleotide (Nusinersen), and displaces the splicing machinery, producing fully functional SMN protein. ${ }^{[43]}$ However, as the treatment effects are transient, repetitive injections may be required to avoid the detrimental developmental effects of the mutation. Thus, a permanent 'once-off' genome-engineering strategy would benefit these patients. However, CRISPR-Cas9 cannot always target specific mutations due to the requirement of a specific NGG recognition sequence. Using a modified version of CRISPR (CRISPR-Cpf1), Zhou et al. ${ }^{[45]}$ were able to identify suitable endonucleases that would allow repair of this mutation, leading to restoration of SMN protein in patient-specific iPSCs.However, it is estimated that only $50 \%$ of patients in SA have the homozygous deletion mutation exhibited globally, due to the complexities of the genomic rearrangements of the region. ${ }^{[46]}$ Furthermore, they reveal frequent deletions of $S M N 2,{ }^{[47]}$ suggesting targeting of SMN2 to restore SMN protein will have limited impact in SA patients. Therefore, if iPSC technology using SA SMA patient-specific cells could be generated, rescue of the mutation could reveal whether these unique patients would benefit from genome engineering targeting the SMN2 gene rearrangement. Spinocerebellar ataxia type 7 (SCA7) offers another opportunity to address an African-specific neurodegenerative issue using allelespecific gene ablation, as it is more common in SA than anywhere else in the world ${ }^{[48]}$ SCA7 is an autosomal dominant disorder caused by an expansion of a trinucleotide repeat producing aggregates of toxic protein in cerebellar glia and the degeneration of Purkinje neurons. With no treatment options, this results in a multitude of defects and lethality within a few years. While gene therapy strategies have been researched with the view to reducing expression of the mutant protein, they are, as with the SMA approach, transient. However, targeting the mutation (an expansion of $>40$ CAG nucleotide repeats) would be inadvisable owing to the number of similar sequences the endonuclease would inadvertently damage in the genome and the normal allele. Since retaining the one correct version of the allele is known to be necessary for normal development, removing just the mutated gene is preferable. Due to a founder effect in black SA patients, a single-nucleotide variant has been found to track with the mutant allele. ${ }^{[49]}$ Thus, using allele-specific genome engineering, targeting of this single nucleotide variant could be used as a unique strategy to destroy the mutated allele in $50 \%$ of SA patients. A similar strategy with in vivo viral vector transduction was deemed feasible for Huntington's disease, another polyglutamine disease. ${ }^{[50]}$

\section{Conclusion}

We chose to showcase diseases where a significant South African component exists, highlighting a few with genetic, societal and/ or environmental impact. There are many other diseases for which genome engineering could be applied in the unique SA context, e.g. cystic fibrosis, beta-thalassaemia and Fanconi anaemia, to name a few. $^{[46]}$ The challenges are significant, not least of which is the cost of such treatments. We must also acknowledge the health burden in SA. Drug-resistant pathogens are considered one of the WHO's most serious health concerns. This, in addition to a lifetime of adverse drug reactions to ARVs, may promote an open-minded attitude towards researching previously considered 'science-fiction' genomeengineering treatments. Furthermore, many of the diseases discussed here have no current treatments, and so, compassionate therapies like the one described for the 6-month-old ALL patient, should be investigated.

In addition, the scientific community must be prudent in addressing ethical concerns related to genome engineering (succinctly discussed here). ${ }^{[51]}$ This is especially important because of recent events in China, where genetically engineered twin girls were born (to an HIVpositive father) with modifications to their CCR5 genes in an attempt to create immunity to HIV. The litany of issues that were raised launched an international outcry; it was unnecessary (sperm-washing can eliminate the virus prior to IVF), incomplete (one of the girls was only heterozygous and therefore not protected from HIV), performed without ethical approval, and has potentially led to their increased susceptibility to other infectious diseases and impairment of neuronal development. ${ }^{[52]}$ The argument for pioneering germline editing lies in advancing the ability to correct many inherited mutations. However, whether germline editing is ready for the clinic is a moot point. The well-established technology of pre-implantation genetic diagnosis already provides a non-invasive method of excluding inheritance of harmful genetic mutations. Therefore, regulatory authorities must develop watertight rules governing the use of genome engineering.

Nonetheless, our country's unique health issues and related genetic diversity necessitate unique solutions. With well-informed debate and scientific oversight, genome engineering could hold the potential to unlocking these solutions. 
Acknowledgements. We thank Professors Amanda Krause and Sue Kidson for helpful discussions.

Author contributions. JS conceptualised the review. EF and JN contributed to the writing of the manuscript.

Funding. CSIR.

Conflicts of interest. None.

1. Aiuti A, Roncarolo MG, Naldini L. Gene therapy for ADA-SCID, the first marketing approval of an ex vivo gene therapy in Europe: Paving the road for the next generation of advanced therapy medicinal products. EMBO Mol Med 2017;9(6):737-740. https://doi.org/10.15252/emmm.201707573

2. Barrangou R. RNA events. Cas9 targeting and the CRISPR revolution. Science 2014;344(6185):707708. https://doi.org/10.1126/science.1252964

3. Chandrasegaran S, Carroll D. Origins of programmable nucleases for genome engineering. J Mol Biol 2016;428(5 Pt B):963-989. https://doi.org/10.1016/j.jmb.2015.10.014

4. Qi LS, Larson MH, Gilbert LA, et al. Repurposing CRISPR as an RNA-guided platform for sequence-specific control of gene expression. Cell 2013;152(5):1173-1183. https://doi.org/10.1016/j. sequence-specific
cell.2013.02.022

5. Maeder ML, Linder SJ, Cascio VM, Fu Y, Ho QH, Joung JK. CRISPR RNA-guided activation of Maeder ML, Linder S,, Cascio VM, Fu Y, Ho QH, Joung JK. CRISPR RNA-guided activation
endogenous human genes. Nat Methods 2013;10(10):977-979. https://doi.org/10.1038/nmeth.2598

endogenous human genes. Nat Methods 2013;10(10):977-979. https://doi.org/10.1038/nmeth.2598
6. Jinek M, Chylinski K, Fonfara I, Hauer M, Doudna JA, Charpentier E. A programmable dual-RNA. Jinek M, Chylinski K, Fonfara I, Hauer M, Doudna JA, Charpentier E. A programmable dual-RNA-
guided DNA endonuclease in adaptive bacterial immunity. Science 2012;337(6096):816-821. https:// guided DNA endonuclease in adap
doi.org/10.1126/science.1225829

7. Dunbar CE, High KA, Joung JK, Kohn DB, Ozawa K, Sadelain M. Gene therapy comes of age. Science 2018;359(6372):pii:eaan4672. https://doi.org/10.1126/science.aan4672

8. Amoasii L, Hildyard JCW, Li H, et al. Gene editing restores dystrophin expression in a canine model of Duchenne muscular dystrophy. Science 2018;362(6410):86-91. https://doi.org/10.1126/science. aau1549

9. Aartsma-Rus A, Fokkema I, Verschuuren J, et al. Theoretic applicability of antisense-mediated exon skipping for Duchenne muscular dystrophy mutations. Hum Mutat 2009;30(3):293-299. https://doi. org/10.1002/humu. 20918

10. Godfrey C, Muses S, McClorey G, et al. How much dystrophin is enough: The physiological consequences of different levels of dystrophin in the mdx mouse. Hum Mol Genet 2015;24(15):4225consequences of different levels of dystrop
4237. https://doi.org/10.1093/hmg/ddv155

11. Reardon S. Leukaemia success heralds wave of gene-editing therapies. Nature 2015;527(7577):146-147. Reardon S. Leukaemia success heralds wave
https://doi.org/10.1038/nature.2015.18737

12. National Cancer Institute. Tisagenlecleucel Approved to Treat Some Lymphomas. 2018. https://www. cancer.gov/news-events/cancer-currents-blog/2018/tisagenlecleucel-fda-lymphoma (accessed 19 March 2019)

13. Yáñez-Muñoz RJ, Grupp SA. CART in the clinic: Drive with care. Gene Ther 2018;25(3):157-161.

14. Brudno JN, Kochenderfer JN. Toxicities of chimeric antigen receptor T cells: Recognition and management. Blood 2016;127(26):3321-3330. https://doi.org/10.1182/blood-2016-04-703751

15. Valton J, Guyot V, Boldajipour B, et al. A versatile safeguard for chimeric antigen receptor T-cell immunotherapies. Sci Rep 2018;8(1):8972. https://doi.org/10.1038/s41598-018-27264-w

16. Dessels C, Alessandrini M, Pepper MS. Factors influencing the umbilical cord blood stem cell industry: An evolving treatment landscape. Stem Cells Transpl Med 2018;7(9):643-650. https://doi. org/10.1002\%2Fsctm.17-0244

17. Thomson JA, Itskovitz-Eldor J, Shapiro SS, et al. Embryonic stem cell lines derived from human blastocysts. Science 1998;282(5391):1145-1147.

18. Takahashi $\mathrm{K}$, Tanabe $\mathrm{K}$, Ohnuki $\mathrm{M}$, et al. Induction of pluripotent stem cells from adult human . Takahashi K, Tanabe K, Ohnuki M, et al. Induction of pluripotent stem cells from adult hu
fibroblasts by defined factors. Cell 2007;131(5):861-872. https://doi.org/10.1016/..cell.2007.11.019

19. Steinbeck JA, Studer L. Moving stem cells to the clinic: Potential and limitations for brain repair
. 19. Steinbeck JA, Studer L. Moving stem cells to the clinic: Potential and limithen
Neuron 2015;86(1):187-206. https://doi.org/10.1016/i.neuron.2015.03.002

Neuron 2015;86(1):187-206. https://doi.org/10.1016/j.neuron.2015.03.002
20. Parmar M, Takahashi J, Studer L, Barker RA. GFORCE-PD still going strong in 2016. NPJ Park Dis 2017;3:16014. https://doi.org/10.1038\%2Fnpjparkd.2016.14

21. Nature. 'Reprogrammed' stem cells implanted into patient with Parkinson's disease. https://www. nature.com/articles/d41586-018-07407-9 (accessed 18 March 2019).

22. Soldner F, Jaenisch R. Medicine. iPSC disease modeling. Science 2012;338(6111):1155-1156. https:// doi.org/10.1126/science. 1227682

23. Patel SJ, Sanjana NE, Kishton RJ, et al. Identification of essential genes for cancer immunotherapy. Nature 2017;548(7669):537-542. https://doi.org/10.1038/nature23477

24. UNAIDS. AIDSinfo. http://aidsinfo.unaids.org/ (accessed 18 March 2019).

25. Birbal S, Dheda M, Ojewole E, Oosthuizen F. Adverse drug reactions associated with antiretroviral therapy in South Africa. Afr J AIDS Res 2016;15(3):243-248. https://doi.org/10.2989/16085906.201 6.1191519
26. McLaren PJ, Carrington M. The impact of host genetic variation on infection with HIV-1. Nat Immunol 2015;16(6):577-583. https://doi.org/10.1038/ni.3147

27. Hütter G, Nowak D, Mossner M, et al. Long-term control of HIV by CCR5 Delta32/Delta32 stem-cell Hutter G, Nowak D, Mossner M, et al. Long-term control of HIV by CCR5 Delta32/Delta32 $\mathrm{s}$ tem-1
transplantation. N Engl J Med 2009;360(7):692-698. https://doi.org/10.1056/NEJMoa0802905

28. Liu Z, Chen S, Jin X, et al. Genome editing of the HIV co-receptors CCR5 and CXCR4 by CRISPR-Cas9 protects CD4+ T cells from HIV-1 infection. Cell Biosci 2017;7:47. https://doi. CRISPR-Cas9 protects CD4+ T cell
org/10.1186\%2Fs $13578-017-0174-2$

29. Ophinni Y, Inoue M, Kotaki T, Kameoka M. CRISPR/Cas9 system targeting regulatory genes of HIV-1 inhibits viral replication in infected T-cell cultures. Sci Rep 2018;8(1):7784. https://doi. org/10.1038/s41598-018-26190-1

30. Wagner TA. Quarter century of anti-HIV CAR T cells. Curr HIV/AIDS Rep 2018;15(2):147-154 https://doi.org/10.1007/s11904-018-0388-x

31. Bella R, Kaminski R, Mancuso P, et al. Removal of HIV DNA by CRISPR from patient blood engrafts in humanized mice. Mol Ther Nucleic Acids 2018;12:275-282. https://dx.doi.org/10.1016\%2F omtn.2018.05.021

32. Bialek JK, Dunay GA, Voges M, et al. Targeted hiv-1 latency reversal using crispr/cas9-derived transcriptional activator systems. PLoS One 2016;11(6):e0158294. https://doi.org/10.1371/journal. pone.0158294

33. Park RJ, Wang T, Koundakjian D, et al. A genome-wide CRISPR screen identifies a restricted set of HIV host dependency factors. Nat Genet 2017;49(2):193-203. https://doi.org/10.1038/ng. 3741

34. Ely A, Moyo B, Arbuthnot P. Progress with developing use of gene editing to cure chronic infection . Ely A, Moyo B, Arbuthnot P. Progress with developing use of gene editing to cure chronic infection
with hepatitis B virus. Mol Ther J Am Soc Gene Ther 2016;24(4):671-677. https://doi.org/10.1038/ with hepatitis

35. Bloom K, Ely A, Mussolino C, Cathomen T, Arbuthnot P. Inactivation of hepatitis B virus replication in cultured cells and in vivo with engineered transcription activator-like effector nucleases. Mol Ther J Am Soc Gene Ther 2013;21(10):1889-1897. https://doi.org/10.1038/mt.2013.170

36. De Buhr H, Lebbink RJ. Harnessing CRISPR to combat human viral infections. Curr Opin Immunol 2018;54:123-129. https://doi.org/10.1016/j.coi.2018.06.002

37. Scott T, Moyo B, Nicholson S, et al. ssAAVs containing cassettes encoding SaCas9 and guide targeting hepatitis B virus inactivate replication of the virus in cultured cells. Sci Rep 2017;7(1):7401 https://doi.org/10.1038/s41598-017-07642-6

38. Mswela M. Violent attacks against persons with albinism in South Africa: A human rights perspective. Afr Hum Rights Law J 2017;17(1):114-133. https://doi.org/10.17159/1996-2096/2017/ v17nla6

39. Kromberg JGR, Bothwell J, Kidson SH, Manga P, Kerr R, Jenkins T. Types of albinism in the black Southern Africa population. East Afr Med J 2012;89(1):20-27.

40. Manga P, Kerr R, Ramsay M, Kromberg JGR. Biology and genetics of oculocutaneous albinism and vitiligo - common pigmentation disorders in southern Africa. S Afr Med J 2013;103(12 Suppl 1):984-988.

41. Baulier E, Garcia Diaz A, Corneo B, Farber DB. Generation of a human ocular albinism type iPSC line, SEIi001-A, with a mutation in GPR143. Stem Cell Res 2018;33:274-277. https://doi. org/10.1016/j.scr.2018.11.016

42. Douglas AGL, Wood MJA. Splicing therapy for neuromuscular disease. Mol Cell Neurosci 2013;56:169-185. https://doi.org/10.1016/j.mcn.2013.04.005

43. Talbot K, Tizzano EF. The clinical landscape for SMA in a new therapeutic era. Gene The 2017;24(9):529-533. https://doi.org/10.1038/gt.2017.52

44. Zetsche B, Gootenberg JS, Abudayyeh OO, et al. Cpfl is a single RNA-guided endonuclease of a clas 2 CRISPR-Cas system. Cell 2015;163(3):759-771. https://doi.org/10.1016/j.cell.2015.09.038

45. Zhou M, Hu Z, Qiu L, et al. Seamless genetic conversion of $\operatorname{smn} 2$ to $s m n 1$ via CRISPR/Cpfl and singlestranded oligodeoxynucleotides in spinal muscular atrophy patient-specific induced pluripoten stem cells. Hum Gene Ther 2018;29(11):1252-1263. https://doi.org/10.1089/hum.2017.255

46. Krause A, Seymour H, Ramsay M. Common and founder mutations for monogenic traits in subSaharan African populations. Annu Rev Genomics Hum Genet 2018;31(19):149-175. https://doi. org/10.1146/annurev-genom-083117-021256

47. Voster E. Determining the molecular basis of spinal muscular atrophy in the black South African population. Doctoral thesis. 2017. http://wiredspace.wits.ac.za/handle/10539/25810 (accessed 10 March 2019)

48. Bryer A, Krause A, Bill P, et al. The hereditary adult-onset ataxias in South Africa. J Neurol Sci 2003;216(1):47-54.

49. Greenberg J, Solomon GAE, Vorster AA, Heckmann J, Bryer A. Origin of the SCA7 gene mutation in South Africa: Implications for molecular diagnostics. Clin Genet 2006;70(5):415-417. https://doi org/10.1111/j.1399-0004.2006.00680.

50. Monteys AM, Ebanks SA, Keiser MS, Davidson BL. CRISPR/Cas9 editing of the mutant Huntingtin allele in vitro and in vivo. Mol Ther J Am Soc Gene Ther 2017;25(1):12-23. https://doi.org/10.1016/j. ymthe.2016.11.010

51. Pepper MS, Pope A, Kling S, Alessandrini M, van Staden W, Green RJ. Ethical considerations in the application of cell and gene therapies in children. S Afr Med J 2018;108(12):1027-1029. https://doi. org/10.7196/SAMJ.2018.v108i12.13585

52. Cyranoski D, Ledford H. Genome-edited baby claim provokes international outcry. Nature 2018;563(7733):607-608. https://doi.org/10.1038/d41586-018-07545-0 